OSU-HEP-02-02

BA-02-04

February 2002

\title{
Family Unification in Five and Six Dimensions
}

\author{
K.S. Babu ${ }^{1}$, S.M. Barr ${ }^{2}$, and Bumseok Kyae ${ }^{2}$ \\ ${ }^{1}$ Department of Physics, Oklahoma State University, \\ Stillwater, OK 74078 \\ ${ }^{2}$ Bartol Research Institute, University of Delaware, \\ Newark, DE 19716
}

\begin{abstract}
In family unification models, all three families of quarks and leptons are grouped together into an irreducible representation of a simple gauge group, thus unifying the Standard Model gauge symmetries and a gauged family symmetry. Large orthogonal groups, and the exceptional groups $E_{7}$ and $E_{8}$ have been much studied for family unification. The main theoretical difficulty of family unification is the existence of mirror families at the weak scale. It is shown here that family unification without mirror families can be realized in simple five-dimensional and six-dimensional orbifold models similar to those recently proposed for $S U(5)$ and $S O(10)$ grand unification. It is noted that a family unification group that survived to near the weak scale and whose coupling extrapolated to high scales unified with those of the Standard model would be evidence accessible in principle at low energy of the existence of small (Planckian or GUT-scale) extra dimensions.
\end{abstract}




\section{Introduction}

\section{Family unification.}

It has long been known that theories with extra dimensions afford ways of breaking gauge symmetries unavailable in $4 d$ theories [1]. Recently, such mechanisms have been applied to the problem of constructing realistic grand unified models [2, 3]. In particular, it has been shown that orbifold compactification of one or two extra dimensions allows symmetries to be broken in such a way as to resolve in an apparently simple fashion two of the thorniest problems of $4 d$ grand unification, namely the doublet-triplet splitting problem and the problem of dimension-5 proton decay operators. In this letter we show that the same ideas can be applied to a problem almost equally old, that of "family unification" ๒1, 5, 6, 7, 8, 9, 10, 11.

The idea of family unification is an extension of the idea of grand unification. Grand unification has two aspects, gauge unification and quark-lepton unification. Gauge unification is the unification of all the Standard Model gauge groups within a simple group, such as $S U(5)$, $S O(10)$, or $E_{6}$. Quark-lepton unification is the idea that quarks and leptons are put together into irreducible multiplets of that simple group. There is complete quark-lepton unification if all the fermions of one family are contained in a single irreducible representation. This is possible in $S O(10), E_{6}$, and larger groups. The idea of family unification has the same two aspects. It involves, first, the existence of a family gauge group that is unified with the Standard Model gauge group into a simple group [4], and, second, the existence of a single irreducible representation of that group that contains the quarks and leptons of all three known families. For example, if there is a family group $S U(3)$, it can be unified with the Standard Model group within $E_{8}$, since $E_{8} \supset E_{6} \times S U(3) \supset S U(3) \times S U(2) \times U(1) \times S U(3)$. At the same time, in $E_{8}$ all three families can be unified into one irreducible representation, the 248. The groups that have been most studied from the point of view of family unification are $O(14)[7], S O(16)$ [8], $S O(18)$ [9], $E_{7}$ [10], and $E_{8}$ [11].

\section{The problem of mirror families.}

The central problem of family unification is that the ordinary $V-A$ families must be accompanied by an equal number of $V+A$ or "mirror" families. For example, the 248 of $E_{8}$ decomposes under $E_{6} \times S U(3)$ into

$$
248 \rightarrow(78,1)+(1,8)+(27,3)+(\overline{27}, \overline{3}) .
$$

Together with three ordinary families, $(\mathbf{2 7}, \mathbf{3})$, one finds three mirror families, $(\overline{\mathbf{2 7}}, \overline{\mathbf{3}})$. Similarly, for the orthogonal groups: a spinor of $S O(10+2 n)$ decomposes under the $S O(10) \times$ 
$S O(2 n)$ subgroup as

$$
2^{(4+n)} \rightarrow\left(\mathbf{1 6}, 2^{(n-1)}\right)+\left(\overline{\mathbf{1 6}}, 2^{(n-1) \prime}\right) .
$$

(For $n$ odd the $2^{(n-1)}$ and $2^{(n-1) \prime}$ are conjugate to each other, whereas for $\mathrm{n}$ even they are each self-conjugate. For a review of spinor representations of orthogonal groups see [5].) One sees that for every $\mathbf{1 6}$ there is a $\overline{\mathbf{1 6}}$.

The fact that family unification generally gives equal numbers of families and mirror families presents the problem of explaining why no mirror families have been observed. The obvious solution would be to give large mass in some way to the mirror families. However, this turns out to be not so easy. While mirror families could have large $S U(2)_{L} \times U(1)_{Y^{-}}$ invariant mass terms that couple them to ordinary families, such terms give mass to equal numbers of families and mirror families and so do not resolve the problem. The only way to give the mirror families large masses without giving large mass to the ordinary families is with $S U(2)_{L} \times U(1)_{Y}$-breaking mass terms. Such masses would have to be at or below the weak-interaction scale, meaning that the mirror families should be observable through radiative effects in precision tests of the electroweak theory. Recent analyses $[12,13]$ conclude that some additional families or mirror families are allowed, with Ref. [12] finding a chisquared minimum for the number of extra families to be somewhere between one and two. Adler argues that even three mirror families may not be completely excluded by present data [14]. However, even if mirror families with masses in the hundred $\mathrm{GeV}$ range are consistent with data, explaining in a natural way why their masses are much larger than the observed quarks and leptons will be a theoretical challenge. No simple and plausible mechanism which attempts this is known.

Given that it is quite difficult to hide mirror families at the weak scale the question arises whether in a model with family unification the mirror families might be banished altogether from the low-energy spectrum. One interesting possibility is that the mirror families are confined at a high scale [6, 5, 15]. Suppose, for example, that the family unification group is $S O(18)$ and breaks down to $S O(10) \times S O(8)$ at a scale $M_{G}$. The quarks and leptons are then in the representations $(\mathbf{1 6}, \mathbf{8})+\left(\overline{\mathbf{1 6}}, \mathbf{8}^{\prime}\right)$. (See Eq. (2).) If at a scale $M_{f a m} \gg M_{W}$ the family group $S O(8)$ breaks to some confining group $H$ under which the $8^{\prime}$ decomposes into only non-singlets, then all of the mirror families are confined. If, on the other hand, 8 contains some singlets in its decomposition under $H$, then some ordinary families will be unconfined and appear in the low-energy spectrum. For instance, there is an $S O(5)$ subgroup of $S O(8)$ under which $\mathbf{8}^{\prime} \rightarrow \mathbf{4}+\overline{\mathbf{4}}$ but $\mathbf{8} \rightarrow \mathbf{5}+\mathbf{1}+\mathbf{1}+\mathbf{1}$, so that if $S O(5)$ confined three families would remain light [6, 5]. Unfortunately, it is doubtful that there exist models of family unification in four-dimensions in which the gauge coupling of $H$ is asymptotically free and thus able to 
confine the mirror families at some dynamically generated high scale. (The beta function for the $S O(5)$ gauge coupling in the example given above is $d g_{5} / d \ln \mu=+\left\{(62 / 3) /\left(16 \pi^{2}\right)\right\} g_{5}^{3}$ in the non-supersymmetric case, with a larger positive value if there is supersymmetry.)

It would seem, therefore, that in four-dimensional theories mirror families cannot be banished from the low-energy theory. However, it can happen in theories with extra dimensions [16, 17, 19]. For example, as was shown in Ref. [16], in Calabi-Yau compactification of heterotic string theory, the $E_{8}$ of the observable sector can be broken down to $E_{6}$ below the compactification scale with a chiral low-energy spectrum of quarks and leptons. In Ref. [17] it was shown that orbifold compactification from ten to four dimensions can leave an $E_{6} \times S U(3)$ subgroup of $E_{8}$ unbroken, with a chiral low energy spectrum which contains some families in multiplets of the $S U(3)$. (In the particular example of that paper there were thirty-six families (in $3 \times(\mathbf{2 7}, \mathbf{3})+27 \times(\mathbf{2 7}, \mathbf{1})$ ) and no mirror families. For early string theory models with three families, see Ref. [18].) Such string theory models have family unification in the sense that all the families of quarks and leptons originate from one $E_{8}$ multiplet of the ten-dimensional theory.

In this paper we show that family unification can be achieved in simple $5 d$ and $6 d$ orbifold models quite similar in spirit to those of [目, 司]. These are "bottom up" models, like those of [2, 3], and no attempt is made to derive them from superstring theory. In the next section, we present simple illustrative models based on both orthogonal groups and $E_{8}$. Before we turn to higher dimensions, however, it may be helpful to briefly review some facts about the family unification groups that have been studied in the literature.

\section{Family unification groups.}

First let us look at the smallest group that has been used for family unification, $O(14)$. We see from Eq. (1) that a spinor of $S O(14)$ decomposes under $S O(10) \times S O(4)$ as $\mathbf{6 4} \rightarrow$ $(\mathbf{1 6}, \mathbf{2})+\left(\overline{\mathbf{1 6}}, \mathbf{2}^{\prime}\right)$. The conjugate spinor decomposes as $\overline{\mathbf{6 4}} \rightarrow\left(\mathbf{1 6}, \mathbf{2}^{\prime}\right)+(\overline{\mathbf{1 6}}, \mathbf{2})$. Thus, a single spinor of $S O(14)$ can only accomodate two families and their mirrors. However, if one takes the group to be $O(14)$ (that is $S O(14)$ together with the parity that transforms the $\mathbf{6 4}$ and $\overline{\mathbf{6 4}}$ into each other) then the irreducible spinor is $\mathbf{1 2 8}(=\mathbf{6 4}+\overline{\mathbf{6 4}})$, which can accomodate four families. Models of family unification based on $O(14)$ have been constructed [7].

The group $S O(16)$ is large enough for family unification, since $\mathbf{1 2 8} \rightarrow(\mathbf{1 6}, \mathbf{4})+(\overline{\mathbf{1 6}}, \overline{\mathbf{4}})$ (and $\left.\mathbf{1 2 8}^{\prime} \rightarrow(\mathbf{1 6}, \overline{\mathbf{4}})+(\overline{\mathbf{1 6}}, \mathbf{4})\right)$. However, its spinors are real, i.e. self-conjugate, so that in the absence of any other symmetry there would be nothing to prevent a mass term of the form $128 \cdot 128$, which would naturally make all the quarks and leptons superheavy. (The same objection applies to $O(14)$.) For this reason most authors have concentrated on the group $S O(18)$ [9], which is the smallest orthogonal group whose spinors are complex and 
large enough to contain at least three families. However, it is possible to avoid the self-mass problem in $S O(16)$ by assuming that the $\mathbf{1 2 8}$ of quarks and leptons is charged under some other group, say a $U(1)$. Interesting $S O(16)$ models using this idea have been constructed [8].

The group $E_{7}$ has an adjoint that under the $S U(5) \times S U(3)$ subgroup decomposes as $\mathbf{1 3 3} \rightarrow(\mathbf{2 4}, \mathbf{1})+(\mathbf{1}, \mathbf{8})+(\mathbf{5}, \mathbf{1})+(\overline{\mathbf{5}}, \mathbf{1})+(\mathbf{1}, \mathbf{1})+(\mathbf{1 0}, \overline{\mathbf{3}})+(\overline{\mathbf{5}}, \mathbf{3})+(\overline{\mathbf{1 0}}, \mathbf{3})+(\mathbf{5}, \overline{\mathbf{3}})$. As with the other groups we have discussed, this has an equal number of families and mirror families. But it also has the interesting peculiarity that some of the fermions in a family transform as 3 of the $S U(3)$ family group, while others transform as $\overline{\mathbf{3}}$. It should be noted also, that the self-mass problem exists for both $E_{7}$ and $E_{8}$ (as it does for $O(14)$ and $S O(16)$ ) since both the $\mathbf{1 3 3}$ of $E_{7}$ and the $\mathbf{2 4 8}$ of $E_{8}$ are real representations.

\section{Family Unification without the Mirror Families}

We start with a simple $S O(16)$ model of family unification in five dimensions in which the low-energy four-dimensional theory has three light families in a triplet of $S U(3)$ and no mirror families.

Following in the footsteps of [2], we consider a five-dimensional theory with the fifth dimension (y) compactified on an $S^{1} /\left(Z_{2} \times Z_{2}^{\prime}\right)$ orbifold. The circumference of $S^{1}$ is $2 \pi R$. $Z_{2}$ reflects $y \rightarrow-y$, and $Z_{2}^{\prime}$ reflects $y^{\prime} \rightarrow-y^{\prime}$, where $y^{\prime}=y+\pi R / 2$. (See Fig. 1.) The orbifold $S^{1} /\left(Z_{2} \times Z_{2}^{\prime}\right)$ can be taken to be the interval $-\pi R / 2 \leq y \leq 0$. The point $y=0$, which we will call $O$, is a fixed point of $Z_{2}$, while the point $y=-\pi R / 2$ (or $y^{\prime}=0$ ), which we will call $O^{\prime}$, is a fixed point of $Z_{2}^{\prime}$. There are branes at the points $O$ and $O^{\prime}$. It is assumed that in the five-dimensional bulk there is an $N=1$ supersymmetric theory with gauge group $S O(16)$ and fields consisting of a vector multiplet in the adjoint (120) and a hypermultiplet in the spinor (128).

The $N=1$ supersymmetry in five dimensions would give $\mathrm{N}=2$ supersymmetry in four dimensions, but the orbifold compactification will break this down to $N=1$. The vector multiplet of the bulk theory splits into a vector multiplet $V^{a}$ and a chiral multiplet $\Sigma^{a}$ of the unbroken $4 d N=1$ supersymmetry. Under the $Z_{2}$ symmetry $V^{a}$ is taken to have parity + and $\Sigma^{a}$ to have parity -. Similarly, the hypermultiplet splits into two chiral multiplets $\Phi$ and $\Phi^{c}$ having opposite $Z_{2}$ parity. The $Z_{2}^{\prime}$ parities are assigned in the following way. The $S O(16)$ adjoint when decomposed under $S O(10) \times S O(6)$ gives $\mathbf{1 2 0} \rightarrow(\mathbf{4 5}, \mathbf{1})+(\mathbf{1}, \mathbf{1 5})+(\mathbf{1 0}, \mathbf{6})$. We assign $Z_{2}^{\prime}$ parity + to the components of the $5 d$ vector multiplet in the $(\mathbf{4 5}, \mathbf{1})+(\mathbf{1}, \mathbf{1 5})$, and $Z_{2}^{\prime}$ parity - to those in the $(\mathbf{1 0}, \mathbf{6})$. This assignment is consistent with the group algebra 


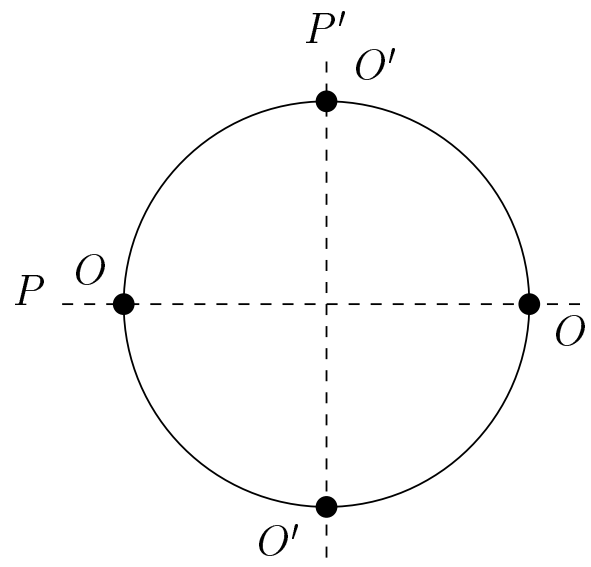

Figure 1: The fifth dimension compactified on an $S^{1} /\left(Z_{2} \times Z_{2}^{\prime}\right)$ orbifold.

of $S O(16)$. The spinor of $S O(16)$ decomposes as $\mathbf{1 2 8} \rightarrow(\mathbf{1 6}, \mathbf{4})+(\overline{\mathbf{1 6}}, \overline{\mathbf{4}})$. Under $Z_{2}^{\prime}$ we take the components of the $5 d$ hypermuliplet that are in $(\mathbf{1 6}, \mathbf{4})$ to have parity + while those in $(\overline{\mathbf{1 6}}, \overline{\mathbf{4}})$ have parity - . The complete $Z_{2} \times Z_{2}^{\prime}$ assignments are shown in Table I.

\begin{tabular}{|c||c|c|c|c|c|c|c|c|c|c|}
\hline & $V_{(\mathbf{4 5}, \mathbf{1})}$ & $V_{(\mathbf{1}, \mathbf{1 5})}$ & $V_{(\mathbf{1 0}, \mathbf{6})}$ & $\Sigma_{(\mathbf{4 5}, \mathbf{1})}$ & $\Sigma_{(\mathbf{1}, \mathbf{1 5})}$ & $\Sigma_{(\mathbf{1 0}, \mathbf{6})}$ & $\Phi_{(\mathbf{1 6}, \mathbf{4})}$ & $\Phi_{(\overline{\mathbf{1 6}}, \overline{\mathbf{4}})}$ & $\Phi_{(\mathbf{1 6}, \mathbf{4})}^{c}$ & $\Phi_{(\overline{\mathbf{1 6}}, \overline{\mathbf{4}})}^{c}$ \\
\hline \hline$Z_{2}$ & + & + & + & - & - & - & + & + & - & - \\
$Z_{2}^{\prime}$ & + & + & - & + & + & - & + & - & - & + \\
\hline
\end{tabular}

Table I. $\left(Z_{2}, Z_{2}^{\prime}\right)$ parity assignments for the vector and hypermultiplets in $S O(16)$.

As explained in many papers [2], only those fields that have $Z_{2} \times Z_{2}^{\prime}$ parity $(+,+)$ can have among their Kaluza-Klein modes one that is constant in the fifth dimension, i.e. a zero mode corresponding to a light field in the four-dimensional effective theory. Fields that have $(+,-),(-,+)$, or $(-,-)$ parities must vanish at the fixed points $O, O^{\prime}$, or both, and therefore have only higher Kaluza-Klein modes, which are superheavy from the $4 d$ point of view. In this model the four-dimensional effective theory will have $N=1$ supersymmetry, gauge group $S O(10) \times S O(6)$, and light fields coming from the bulk matter consisting of vector multiplets in $(\mathbf{4 5}, \mathbf{1})$ and $(\mathbf{1}, \mathbf{1 5})$ and a chiral multiplet in $(\mathbf{1 6}, \mathbf{4})$. This cannot be the complete effective low-energy theory since it has an $S O(6)^{3}$ gauge anomaly. One must take into account also fields that "live" on the branes at $O$ and $O^{\prime}$.

On the brane at $O$ there is the full $S O(16)$ gauge symmetry, but on the brane at $O^{\prime}$ there is only an $S O(10) \times S O(6)$. It is not that $S O(16)$ is spontaneously broken on the $O^{\prime}$ brane, but rather that there never was any gauge symmetry on $O^{\prime}$ except $S O(10) \times S O(6)$. (At $O^{\prime}$ quantities that are odd under $Z_{2}^{\prime}$ have to vanish, including the gauge parameters $\xi^{a}\left(x^{\mu}, y\right)$ 
for those generators $\lambda^{a}$ in the coset $S O(16) /(S O(10) \times S O(6))$.) Consequently, in the $4 d$ theory on the brane at $O^{\prime}$ only complete multiplets of $S O(10) \times S O(6)$ but not $S O(16)$ need appear. Let us suppose then that on the $O^{\prime}$ brane one has quark and lepton chiral multiplets in $(\overline{\mathbf{1 6}}, \mathbf{1})+2 \times(\mathbf{1}, \overline{\mathbf{1 0}})$ and Higgs chiral multiplets in $(\mathbf{1}, \mathbf{4})+(\mathbf{1}, \overline{\mathbf{4}})$. (We will denote the Higgs multiplets henceforth with subscript ' $H$ '.) The low-energy theory then has altogether the following chiral multiplets: $(\mathbf{1 6}, \mathbf{4})+(\overline{\mathbf{1 6}}, \mathbf{1})+2 \times(\mathbf{1}, \overline{\mathbf{1 0}})+(\mathbf{1}, \mathbf{4})+(\mathbf{1}, \overline{\mathbf{4}})$. This set has no gauge anomalies. (The $S O(6)^{3}$ anomaly of a $\overline{\mathbf{1 0}}$ is -8 times that of a $\mathbf{4}$, so the total $S O(6)^{3}$ anomaly of the above set is $16(1)+2(-8)=0$.)

We assume that the $(\mathbf{1}, \mathbf{4})_{H}+(\mathbf{1}, \overline{\mathbf{4}})_{H}$ obtain vacuum expectation values at some scale $M_{\text {family }} \gg M_{W}$ that breaks $S O(6)(=S U(4))$ down to $S U(3)$. Then a term of the form $(\mathbf{1 6}, \mathbf{4})(\overline{\mathbf{1 6}}, \mathbf{1})(\mathbf{1}, \overline{\mathbf{4}})_{H}$ on the $O^{\prime}$ brane will give mass to the $(\overline{\mathbf{1 6}}, \mathbf{1}) V+A$ family and one of the four $V-A$ families to leave three light families in a $(\mathbf{1 6}, \mathbf{3})$ of $S O(10) \times S U(3)$. Note that the scale $M_{\text {family }}$ can be anything from the weak scale up to the compactification scale.

There still remain the questions of how $S O(10)$ breaks down to the Standard Model group in a satisfactory way, how the electroweak symmetry is broken, how realistic masses arise for the quarks and leptons, and how the $S U(3)$ family symmetry breaks. These breakings can all be accomplished through the ordinary Higgs mechanism by Higgs multiplets living on the $O^{\prime}$ brane in suitable representations of $S O(10) \times S O(6)$. We know, for example, that breaking of $S O(10)$ down to the Standard Model can be achieved in $4 d$ SUSY GUTs in such a way as to have natural doublet-triplet splitting [20] and also suppression of dimension-5 proton decay operators (i.e. those coming from colored Higgsino exchange) [21]. It would be interesting to attempt to use orbifold compactification to break $S O(10)$ down to the Standard Model as well, as in [3]. However, we do not pursue that more ambitious goal in this paper.

What we have shown in this simple example is that models can be constructed in which the three light families come from a single irreducible representation of a simple group here a hypermultiplet in the bulk $S O(16)$ theory, and in which that simple group comprises both the Standard Model gauge symmetries and a family gauge symmetry that survives in the four-dimensional theory, perhaps even down to low energy. It is clear that one may construct $S O(18)$ models in a similar way. However, the analogous orbifold breaking to $S O(10) \times S O(8)$ would give eight families coming from the hypermultiplet in the bulk. One would therefore have to introduce five mirror families on the $O^{\prime}$ brane, and a correspondingly large number of Higgs on the brane to mate the mirror families with the ordinary ones. It would be interesting to know if a more economical and compelling way to obtain three light families from $S O(18)$ using orbifold compactifications could be found.

We see, then, that the orthogonal groups can accomodate three light families, but they 
do not naturally prefer three light families. If we wish to explain the fact that there are three light families, the group $E_{8}$ seems more promising, given that $E_{6} \times S U(3)$ is a maximal subgroup and that the fundamental representation of $E_{8}$ contains a $(\mathbf{2 7}, \mathbf{3})$ under that subgroup, as shown in Eq. (1).

It is easy to see that orbifolds with $Z_{2}$ symmetries as we have been considering up to now are not adequate to split the $(\overline{\mathbf{2 7}}, \overline{\mathbf{3}})$ mirror families from the $(\mathbf{2 7}, \mathbf{3})$ families in the 248. For a $Z_{2}$ parity to accomplish this, the $(\overline{\mathbf{2 7}}, \overline{\mathbf{3}})$ and $(\mathbf{2 7}, \mathbf{3})$ would have to have opposite parity. Suppose, then, we imagine the parity of $(\mathbf{2 7}, \mathbf{3})$ to be + and that of $(\overline{\mathbf{2 7}}, \overline{\mathbf{3}})$ to be - . This would be inconsistent with the group algebra of $E_{8}$. The 248 generators of $E_{8}$ fall into $(\mathbf{7 8}, \mathbf{1})+(\mathbf{1}, \mathbf{8})+(\mathbf{2 7}, \mathbf{3})+(\overline{\mathbf{2 7}}, \overline{\mathbf{3}})$. The commutator of two $(\mathbf{2 7}, \mathbf{3})$ generators gives a $(\overline{\mathbf{2 7}}, \overline{\mathbf{3}})$ generator. Similarly, the commutator of two $(\overline{\mathbf{2 7}}, \overline{\mathbf{3}})$ generators gives a $(\mathbf{2 7}, \mathbf{3})$ generator. Consequently the gauge fields in $(\mathbf{2 7}, \mathbf{3})$ and $(\overline{\mathbf{2 7}}, \overline{\mathbf{3}})$ must both have + parity. That implies that the families and mirror families have the same parity.

In order to eliminate the mirror families from the $4 d$ theory in $E_{8}$ there must be an orbifold with at least a $Z_{3}$ symmetry. The point is that under a $Z_{3}$ the generators of $E_{8}$ can transform as follows consistently with the group algebra:

$$
\begin{aligned}
& \lambda_{(78,1)} \longrightarrow \lambda_{(78,1)} \\
& \lambda_{(1,8)} \longrightarrow \lambda_{(1,8)} \\
& \lambda_{(27,3)} \longrightarrow \omega \lambda_{(27,3)} \\
& \lambda_{(\overline{27}, \overline{3})} \longrightarrow \omega^{2} \lambda_{(\overline{27}, \overline{3})},
\end{aligned}
$$

where $\omega=e^{2 \pi i / 3}$. Such a $Z_{3}$ can be used to split the families from the mirror families in a 248, as will be seen.

Consider a six-dimensional theory in which the extra two dimensions $\left(x^{5}\right.$ and $\left.x^{6}\right)$ are compactified on an orbifold with a $Z_{3}$ symmetry. The construction of such an orbifold is shown in Fig. 2. One starts with a torus $T^{2}$ defined by identifying the points $z \equiv x^{5}+i x^{6}$ with the points $z+1$ and $z+\omega$. This torus is shown as the large parallelogram in Fig. 2. Under a rotation by $2 \pi / 3$ (i.e. $z \rightarrow \omega z$ ) one sees that the regions labelled 1 get mapped into other regions labelled 1 , and that similarly 2 gets mapped into 2,3 into 3 , and 4 into 4 . The $T^{2} / Z_{3}$ orbifold obtained from $T^{2}$ by identifying $z$ with $\omega z$ thus has as its fundamental region the smaller parallelogram $a b a^{\prime} c$. This orbifold has a $Z_{3}$ symmetry, under which $a, b$, and $c$ are inequivalent fixed points. (The point $a^{\prime}$ is identified with $a$ ). (The orbifold used in [17] is the six-dimensional generalization of this simple two-dimensional orbifold and has 


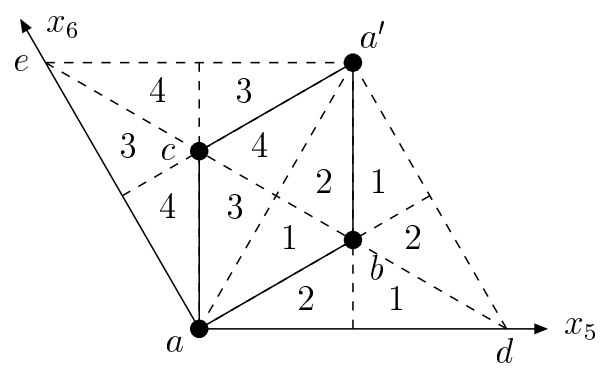

Figure 2: The two extradimensions $x_{5}$ and $x_{6}$ compactified on an $S^{1} / Z_{3}$ orbifold. The regions with the same numbers are identified each other. $a, b$, and $c$ are fixed points of $Z_{3}$, and the parallelogram $a b a^{\prime} c$ is the fundamental region.

27 fixed points.)

Instead of using one discrete symmetry to break supersymmetry down to $N=1$ and another discrete symmetry to break the gauge group as before, we will use the same $Z_{3}$ to break both. In the six-dimensional bulk we take the theory to have $N=1$ supersymmetry and gauge group $E_{8}$. (One need not worry about cancellation of gauge anomalies since the $E_{8}$ box anomaly in six dimensions vanishes up to "reducible" pieces that can be cancelled by the Green-Schwarz mechanism [22].) We take the matter in the bulk to consist of a vector multiplet in the 248. A $6 d$ vector multiplet decomposes, under the $4 d N=1$ supersymmetry, into a vector multiplet $V^{a}$ and a chiral multiplet $\Sigma^{a}$. The index $a$ labels the 248 generators of $E_{8}$, which may be classified under the $E_{6} \times S U(3)$ subgroup as shown in Eq. (1). The fields in $V^{a}$ are assumed to transform under $Z_{3}$ in the same way as the corresponding generators, which is shown in Eq. (3). The fields in $\Sigma^{a}$ transform in the same way except with an additional factor of $\omega^{2}$. The $Z_{3}$ transformation properties of the fields are given in Table II.

\begin{tabular}{|c||c|c|c|c|c|c|c|c|}
\hline & $V_{(\mathbf{7 8}, \mathbf{1})}$ & $V_{(\mathbf{1}, \mathbf{8})}$ & $V_{(\mathbf{2 7}, \mathbf{3})}$ & $V_{(\overline{\mathbf{2 7}}, \overline{\mathbf{3}})}$ & $\Sigma_{(\mathbf{7 8}, \mathbf{1})}$ & $\Sigma_{(\mathbf{1}, \mathbf{8})}$ & $\Sigma_{(\mathbf{2 7}, \mathbf{3})}$ & $\Sigma_{(\overline{\mathbf{2 7}}, \overline{\mathbf{3}})}$ \\
\hline \hline$Z_{3}$ & 1 & 1 & $\omega$ & $\omega^{2}$ & $\omega^{2}$ & $\omega^{2}$ & 1 & $\omega$ \\
\hline
\end{tabular}

Table II. $Z_{3}$ transformation properties of the vector and chiral multiplets in $E_{8}$.

Only those component fields that transform trivially under $Z_{3}$ will have zero modes. Thus, the bulk matter contributes the following fields to the $4 d$ low energy theory: vector multiplets in $(\mathbf{7 8}, \mathbf{1})$ and $(\mathbf{1}, \mathbf{8})$ containing the gauge fields and gaugino fields of $E_{6}$ and $S U(3)$, and a chiral multiplet in $(\mathbf{2 7}, \mathbf{3})$ containing three families of quarks and leptons. This cannot be the whole story as the $4 d S U(3)^{3}$ gauge anomaly must be cancelled. This can be done by chiral multiplets living on one or more of the branes at the fixed points $a$, $b$, and $c$. For example, the $S U(3)^{3}$ anomaly would be cancelled by twenty-seven $(\mathbf{1}, \overline{\mathbf{3}})$ or by 
a single $(\mathbf{1}, \overline{\mathbf{1 0}})$ of $E_{6} \times S U(3)$ on one of the branes. It should be noted that on the branes there is only an $E_{6} \times S U(3)$ symmetry to start with, so that it is only necessary to have complete multiplets of that group rather than of the bulk gauge group $E_{8}$. Again, as in the previous example, we can introduce various (vectorlike) Higgs representations on the branes to break the symmetry down to that of the Standard Model. Such model-building details are beyond the scope of this paper. But we see no obstacle to constructing a fully realistic model in this way.

\section{Family Unification as an indicator of Extra Dimen- sions}

As we have seen, family unification involves the idea that all three known families reside within one irreducible representation of some simple group. This group contains not only the Standard Model gauge groups, but generally a family gauge group. In many cases this family gauge group contains an $S U(3)$ subgroup (or is an $S U(3)$ ) under which the three families form a triplet. The family gauge group could be broken at very high scales, either by an ordinary $4 d$ Higgs field or by the orbifold compactification. But it is also quite possible that a local family symmetry, perhaps an entire $S U(3)$, survives down to "low scale", by which we mean here something in the hundred $T e V$ range.

If family unification is realized in Nature and the family group survives down to low energy the interesting possibility would exist, at least in principle even if quite difficult in practice, to infer the existence of near-Planckian extra dimensions from experiments done at "low energy".

Let us call the set of representations of $G_{S M}=S U(3)_{c} \times S U(2)_{L} \times U(1)_{Y}$ that make up one family of left-handed fermions $\mathbf{F}$, and suppose for specificity that the low energy family symmetry is $S U(3)$ (though most of what we will say applies as well to other groups, such as $S O(3))$. Typically, in models with family unification the known quarks and leptons are in $(\mathbf{F}, \mathbf{3})$ of $G_{S M} \times S U(3)_{f a m}$. (It is also possible that some of the left-handed fermions of a family are in $\mathbf{3}$ and some in $\overline{\mathbf{3}}$ of $S U(3)_{\text {fam }}$, as we saw in the case of family unification in the adjoint of $E_{7}$. What we say below applies to such cases as well.)

If $S U(3)_{f a m}$ is broken at a scale $M_{\text {fam }}$ near enough to the weak scale to be accessible to experiment, then one might be able eventually to do two things: first, to find out what representations of the family group the quarks and leptons are in; and, second, to measure the $S U(3)_{\text {fam }}$ gauge coupling $g_{\text {fam }}$ and extrapolate it to high energies to see whether it unifies

with the Standard Model couplings. Suppose that this is done and it is found that the quarks 
and leptons of the Standard Model are indeed in $(\mathbf{F}, \mathbf{3})$, with no light mirror families, and that the four gauge couplings do indeed unify. What would one be able reasonably to infer from this?

First, the evidence for the unification of the four gauge groups (into one simple group or a product of identical simple groups related by a discrete symmetry) would then be compelling. The unification that we now see of the three Standard Model gauge couplings might be an accident, since it really involves only one non-trivial condition being satisfied. However, the accidental unification of four couplings at one point could hardly be dismissed as accidental.

One would therefore conclude that at a high scale some unified group $G$ broke down to $G_{S M} \times S U(3)_{f a m}$, and that some anomaly free set of representations of $G$ broke into $(\mathbf{F}, \mathbf{3})$ plus some additional multiplets that were vectorlike under $G_{S M}$. (They would have to be vectorlike under $G_{S M}$ in order not to have been seen at the weak scale.) The question that would then confront theorists is whether such a breaking could take place in the context of a theory with only four spacetime dimensions. The answer seems to be no.

We have seen that groups such as $S O(16), S O(18)$, and $E_{8}$ have representations that contain $(\mathbf{F}, \mathbf{3})$ of $G_{S M} \times S U(3)$, but these are always accompanied by mirror families, and in four dimensions there does not seem to be any way to push these mirror families to very large scales. The unitary groups, in particular $S U(N)$ with $N \geq 8$, also can break down to $G_{S M} \times S U(3)$ giving Standard Model multiplets that are in triplets of $S U(3)$, but one never gets simply three families plus pieces that are vectorlike under $G_{S M}$. For example consider $S U(8)$ with quarks and leptons in $\psi^{[\alpha \beta \gamma]}+\psi_{[\alpha \beta]}+\psi_{\alpha}$. Under the $S U(5) \times S U(3)$ subgroup these do give $(\mathbf{1 0}, \mathbf{3})+(\overline{\mathbf{5}}, \overline{\mathbf{3}})$, but these come together with $2(\overline{\mathbf{1 0}}, \mathbf{1})+(\mathbf{5}, \overline{\mathbf{3}})+(\overline{\mathbf{5}}, \mathbf{1})+(\mathbf{1}, \mathbf{3})+$ $(\mathbf{1}, \overline{\mathbf{3}})+(\mathbf{1}, \mathbf{1})$, which are chiral under $G_{S M}$.

The closest that one seems to be able to come is with the group $S U(5) \times S U(5)$, with the two gauge couplings forced to be equal by a discrete symmetry that interchanges the two $S U(5)$ 's. Consider the following anomaly-free set of fermions: $(\mathbf{1 0}, \overline{\mathbf{5}})+(\overline{\mathbf{5}}, \mathbf{1 0})+2(\overline{\mathbf{1 0}}, \mathbf{1})+$ $2(\mathbf{1}, \overline{\mathbf{1 0}})+7(\mathbf{5}, \mathbf{1})+7(\mathbf{1}, \mathbf{5})$. Under the $S U(5) \times S U(3)$ subgroup this gives: $(\mathbf{1 0}, \overline{\mathbf{3}})+(\overline{\mathbf{5}}, \overline{\mathbf{3}})$ together with $2(\overline{\mathbf{5}}, \mathbf{3})+6(\mathbf{5}, \mathbf{1})$ and other pieces that are vectorlike under $S U(5) \times S U(3)$. This indeed gives $(\mathbf{F}, \overline{\mathbf{3}})$ plus pieces that are vectorlike under $G_{S M}$. However, as one can see, those pieces include six $\overline{\mathbf{5}}+\mathbf{5}$ of $S U(5)$ that cannot get mass above $M_{f a m}$ because they are chiral under $S U(3)_{\text {fam }}$. In a supersymmetric theory this would make the couplings blow up below the unification scale.

On the other hand, we have seen that in theories with extra dimensions one can end up with three families in triplets of an $S U(3)$ family gauge symmetry whose coupling unifies at high scales with the Standard Model gauge couplings, without there being any light mirror 
families. Such a situation, if it is found, would be a telltale sign at low energy of a theory with extra dimensions at very high scales not directly accessible to experiment.

\section{Acknowledgments}

The work of KSB is supported in part by DOE Grant $\sharp$ DE-FG03-98ER-41076, a grant from the Research Corporation and by DOE Grant $\sharp$ DE-FG02-01ER-45684. The work of SMB and BK is supported in part by DOE Grant $\sharp$ DE-FG02-91ER-40626.

\section{References}

[1] J. Scherk and J.H. Schwarz, Phys. Lett. B82, 60 (1979); Y. Hosotani, Phys. Lett. B126, 309 (1983); ibid. B129, 193 (1983); P. Candelas, G.T. Horowitz, A. Strominger, and E. Witten, Nucl. Phys., B258, 46 (1985); E. Witten, Nucl. Phys. B258, 75 (1985).

[2] Y. Kawamura, Prog. Theor. Phys. 105, 691 (2001); ibid., 999 (2001); G. Altarelli and F. Feruglio, Phys. Lett. B511, 257 (2001); L.J. Hall and Y. Nomura, Phys. Rev. D64, 055003 (2001); A. Kobakhidze, Phys. Lett. B514, 131 (2001); A. Hebecker and J. March-Russell, Nucl. Phys. B613, 3 (2001); H.D. Kim, J.E. Kim, and H.M. Lee, hep-ph/0112094.

[3] T. Akasi, W. Buchmüller, and L. Covi, hep-ph/0108021; L.J. Hall, Y. Nomura, T. Okui, and D. Smith, Phys. Rev. D65, 035008 (2002); R. Dermíšek and A. Mafi, Phys. Rev. D65, 055002 (2002).

[4] P. Ramond, "The Family Group in Grand Unified Theories", invited talk at the Sanibel Symposia, 1979 (reissued as ArXiv: hep-ph/9809459).

[5] F. Wilczek and A. Zee, Phys. Rev. D25, 553 (1982).

[6] M. Gell-Mann, P. Ramond, and R. Slansky, in Supergravity, ed. P. van Nieuwenhuizen and D.Z. Freedman (North Holland, Amsterdam, 1979).

[7] H. Sato, Phys. Lett. B101, 233 (1981); Phys. Rev. Lett. 45, 1997 (1980); M. Ida, Y. Kayama, and T. Kitazoe, Prog. Theor. Phys. 64, 1745 (1980); A. Masiero, M. Roncadelli, and T. Yanagida, Phys. Lett. B117, 291 (1982).

[8] P. Arnold, Phys. Lett. B149, 473 (1984). 
[9] F. Wilczek and A. Zee, Phys. Rev. D25, 553 (1982); R.N. Mohapatra and B. Sakita, Phys. Rev. D21, 1062 (1980); G. Senjanovic, F. Wilczek, and A. Zee, Phys. Lett. B141, 389 (1984); J. Bagger and S. Dimopoulos, Nucl. Phys. B244, 247 (1984).

[10] M. Koca, Phys. Lett. B107, 73 (1981); T. Kugo and T. Yanagida, Phys. Lett. B134, 313 (1984).

[11] I. Bars and M. Günaydin, Phys. Rev. Lett. 45, 859 (1980); S.E. Konshtein and E.S. Fradkin, Pis'ma Zh. Eksp. Teor. Fiz. 32, 575 (1980 [English transl.: JETP Lett. 32, 557 (1981)]; M. Koca, Phys. Lett. B107, 73 (1981); C.L. Ong, Phys. Rev. D31, 3271 (1985); W. Buchmüller and O. Napoly, Phys. Lett. B163, 161 (1985); K. Itoh, T. Kugo, and H. Kunitomo, Prog. Theor. Phys. 75, 386 (1986); S.M. Barr, Phys. Rev. D37, 204 (1987); U. Ellwanger, Nucl. Phys. B536, 46 (1991).

[12] V.A. Novikov, L.B. Okun, A.N. Rozanov, and M.I. Vysotski, hep-ph/0111028.

[13] H.-J. He, N. Polonsky, and S. Su, hep-ph/0102144.

[14] S.L. Adler, hep-ph/0201009.

[15] G. Triantaphyllou, Eur. Phys. J. C10, 703 (1999).

[16] P. Candelas, G.T. Horowitz, A. Strominger, and E. Witten, Nucl. Phys., B258, 46 (1985).

[17] L.J. Dixon, J.A. Harvey, C. Vafa, and E. Witten, Nucl. Phys. B261, 678 (1985); ibid. 274, 285 (1986).

[18] L.E. Ibanez, H.P. Nilles, and F. Quevedo, Phys. Lett. B187, 25 (1987); L.E. Ibanez, J.E. Kim, H.P. Nilles, and F. Quevedo, Phys. Lett. B191, 282 (1987); L.E. Ibanez, J. Mas, H.P. Nilles, and F. Quevedo, Nucl. Phys. B301, 157 (1988); B.R. Greene, K.H. Kirklin, P.J. Miron, and G.G. Ross, Nucl. Phys. B278, 667 (1986); ibid. 292, 606 (1987).

[19] G. Triantaphyllou and G. Zoupanos, Phys. Lett., B489, 420 (2000).

[20] K.S. Babu and S.M. Barr, Phys. Rev. D48, 5354 (1993).

[21] K.S. Babu and S.M. Barr, hep-ph/0201130.

[22] N. Borghini, Y. Gouverneur, and M.H.G. Tytgat, hep-ph/0108094; A. Hebecker and J. March-Russell, hep-ph/0107039. 